\title{
Chronische Erkrankungen der oberen Luftwege: Wann allergisch bedingt?
}

\section{Die Beschwerden von Patienten mit chronisch-rezidivierenden Infekten der oberen Atemwege sind nicht selten auf eine Allergie zurückzuführen. Eine systematische und parallele Anwendung verschiedener diagnostischer Möglichkeiten hilft bei der allergologischen Diagnosefindung.}

C hronische Erkrankungen der oberen Luftwege imponieren mit einer unspezifischen Symptomatik wie Schwellung, Sekretion, Juckund Niesreiz, Hypo- oder Anosmie und Gesichts- oder Kopfschmerz. Keinesfalls kann von einem einzelnen Symptom auf die Ursache geschlossen werden. Neben anatomischen Veränderungen kommen infektiöse oder hyperreaktive Erkrankungen wie allergische, arzneimittelbedingte, nervalreflektorische, irritativ toxische, endokrine, postinfektiöse oder idiopathische Formen in Frage.

Eine systematische und parallele Anwendung verschiedener diagnostischer Möglichkeiten hilft, eine Allergie als Ursache der Symptome ausfindig zu machen. Dabei ist zunächst $\mathrm{zu}$ berücksichtigen, daß ein Symptom keinesfalls einzeln, sondern auch weitere Symptome, deren situatives, örtliches und zeitliches Auftreten sowie Begleiterkrankungen $\mathrm{zu}$ betrachten sind.

So ergeben sich z.B. folgende Verdachtsdiagnosen:

- Pollenallergie bei saisonalem Auftreten der Beschwerden,

- Hausstaubmilbenallergie bei nächt- licher und morgendlicher Häufung der Beschwerden sowie Beschwerdefreiheit an der See und in den Bergen,

- Tierallergie bei Auslösung der Beschwerden durch Tierkontakt und

- Nahrungsmittelallergie bei Auftreten von Beschwerden nach Genuß bestimmter Nahrungsmittel.

Für die Differentialdiagnose nichtallergischer hyperreaktiver Erkrankungen sind außerdem Informationen über den Gebrauch von Arzneimitteln (vor allem Acetylsalicylsäure), Kontakt mit irritativ toxischen Substanzen sowie über mögliche endokrine Störungen von Bedeutung.

Das größte Problem bei der Dia- gnose allergischer Erkrankungen stellen Mischbilder verschiedener Allergien und die Entwicklung zusätzlicher unspezifischer Hyperreaktivität bei Allergikern dar. Diese führen zu atypischen Beschwerdebildern und Allergietestergebnissen.

Bei der Auswertung von Allergietests ist $\mathrm{zu}$ beachten, daß positive Allergietests nur in Zusammenhang mit Anamnese und klinischem Bild richtig $\mathrm{zu}$ interpretieren sind. Intradermale Testungen besitzen die größte Sensitivität, jedoch eine schlechtere Spezifität mit häufigeren falsch positiven Ergebnissen als Pricktests. Die klinische Relevanz fraglicher Allergene sollte mit Hilfe von Provokationstests am Erfolgsorgan überprüft werden, um Fehlinterpretationen $\mathrm{zu}$ vermeiden. Bei Verdacht auf das Vorliegen einer Lebensmittelallergie empfiehlt sich vielfach die Durchführung einer Eliminationsdiät mit nachfolgender Reexposition gegenüber den in Frage kommenden Allergenen.

Verfahren der zytologischen Diagnostik dienen der Unterscheidung zwischen infektiösen Erkrankungen der Nasen- und Nasennebenhöhlenschleimhaut und hyperreaktiven $\mathrm{Re}$ aktionen.

(jn)

Quelle: Vortrag von R. Mösges (Köln) anläßlich des 25. Kongresses des Ärzteverbandes Deutscher Allergologen, Göttingen 1998.

\section{HNO-Beschwerden: Vom Symptom zur Diagnose}

Symptom
Einseitige Beschwerden
Beidseitige Beschwerden
Alternierende Nasenatmungsbehinderung
Rhinorrhoe
- helles, oft dünnflüssiges Sekret
- grüngelblicher zäher Schleim
- wäßrige Sekretion zusammen mit Juckreiz
- einseitige wäßrige Sekretion
(vor allem nach vorangegangenen
Schädeltraumata oder
Nasennebenhöhlenoperationen)
Riechverlust
- Intermittierender Riechverlust
- Persistierender Riechverlust

typische Ursache

strukturelle Veränderungen der Nase wie Septumdeviationen, Fremdkörper oder Tumoren

funktionelle Veränderungen der Nasenschleimhaut oder Polyposis nasi entspricht dem normalen physiologischen nasalen Zyklus

sekretorische oder exkretorische Störung bei allergischer und nichtallergischer Hyperreaktivität, infektiöser Rhinitis und Sinusitis

hyperreaktive oder virale Erkrankung

bakterielle Infektionen

allergische und andere hyperreaktive Erkrankungen.

Rhinoliquorrhoe

Beteiligung von Ethmoid oder Nasendach, aber keine sichere Diagnose typisch für Rhinitis allergica, infectiosa oder medicamentosa

Polyposis nasi, atrophische Rhinitis oder immunologische Erkrankungen (z.B. Wegenerscher Granulomatose, Sarkoidose) 